to a submarine existence. The taxonomic distribution of the genera mentioned, and others, is such that we may conclude that many saldid genera are exhibiting parallel trends towards a partial or more nearly complete aquatic life.

An obvious specialization of Aepophilus is the presence of bubble-retaining areas on the pronotum, hemelytra and sternal region, but no plastron has been detected. The red coloration is paralleled by that of Aepopsis robinii, a carabid living in similar situations and often collected with Aepophilus ${ }^{8}$; this too has evolved from a group of predominantly black insects. In addition to the structural modifications, there are behavioural ones ; for example, the bugs become immobile when suddenly submerged. Some simple experiments have demonstrated the value of the modifications: the marine-bug can survive at least $23 \frac{1}{2} \mathrm{hr}$. in still sea water, at least $49 \mathrm{hr}$. in running sea water; bugs kept in still sea water for $18 \mathrm{hr}$. were active immediately on release, those kept in boiled sea water for a similar period took about $30 \mathrm{~min}$. to resume activity. Further work is in progress.

44 Abbey Road,

London, N.W.8.

Dennis Leston

${ }^{2}$ Puton, A., "Synopsis des Hémiptères Hétéroptères de France", 1 (Paris, 1879).

2 Bergroth, E., Ent. Mon. Mag., 35, 282 (1899).

' Kirkaldy, G. W., Canad. Ent., 40, 357 (1908).

${ }^{4}$ Reuter, O. M., Acta Soc. Sci. Fenn., 37 (3), 1 (1910).

${ }^{5}$ Pruthi, H. S., Trans. Ent. Soc. Lond., 127 (1925).

${ }^{8}$ Leston, D., and Haskell, P. T. (in preparation).

7 Brown, E. S., J. Anim. Ecol., 17, 180 (1948).

${ }^{3}$ Morton, J. E., J. Mar. Biol. Assoc.. 33, 187 (1954).

\section{Transmission of Infra-red Light in Turbid and Clear Sugar Solutions}

A WELL-FILTERED coloured sugar solution (in water) has an extinction-curve falling with increasing wave-length of light from near ultra-violet to red. Using as reference a clear, colourless sugar solution of the same concentration as the sample, it is possible to extend the observations in the range of near infrared without interference from the strong absorption bands of water at about 980 and $1,175 \mathrm{~m} \mu$. The extinction of the coloured sample will then show a continuous decrease with increasing wave-length up to a range of $1,000-1,100 \mathrm{~m} \mu$, where a flat minimum occurs. At still longer wave-lengths, the extinction rises again. The minimum extinction is very low compared with the optical density for visible light.

The same coloured solution, containing suspended particles (producing turbidity), shows the same behaviour, but naturally has a higher light attenuation than the filtered one at every wave-length. For solutions in the concentration-range common in sugar factories, the difference in optical density between unfiltered and clear-filtered solution is practically the same in the range $500-1,500 \mathrm{~m} \mu$.

Thus the presence of turbid matter can be indicated even in very dark solutions (for example, thick juices in sugar-beet factories and refinery liquors) simply by measurement of the transmission for light of about $1,050 \mathrm{~m} \mu$ in a continuously flowing by-pass stream of the sample.

Unlike the procedure necessary for commonly accepted methods with the same purpose, the 'infrared method' does not involve any special preparation of the sample.

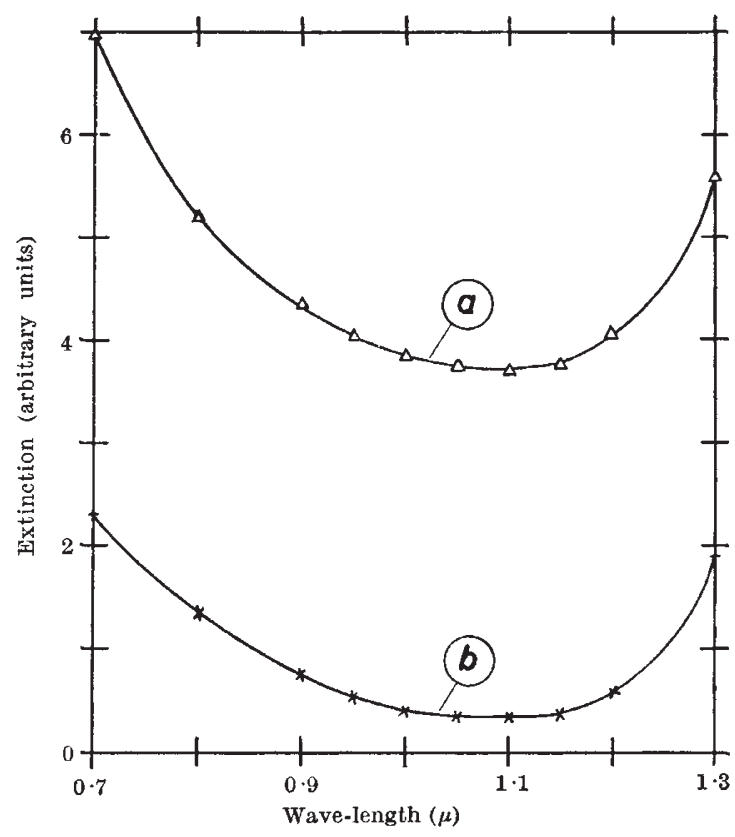
Fig. 1(a). Unfiltered sample of thick juice of sugar beet; $(b)$ clear-
filtered sample of the same juice

The phenomenon is exemplified in Fig. 1, showing a detail of typical extinction curves for one turbid and one clear-filtered sample of the same thick juice of sugar beet. The extinction value at $1,050 \mathrm{~m} \mu$ for the filtered sample is in this case about $1 / 100$ of the value at $463 \mathrm{~m} \mu$.

A full report will be published elsewhere. I wish to thank Mr. R. Lepik for valuable assistance in the investigation.

Åke Birch-IENSEN

Laboratory for Sugar Technology,

Swedish Sugar Corporation,

Arlöv, Sweden.

April 20.

\section{Setting of Gypsum Plaster}

THE initial thickening of slurries of hemi-hydrate type gypsum plasters has been considered by many workers to be due to a colloidal mechanism, whereas the further hardening and final setting have always been attributed to a crystallization process. Recent work supporting the colloidal theory is that of Fischer ${ }^{1}$, while still later Becherer and Fiedler ${ }^{2}$ have attributed the initial thickening of plaster slurries to the action of capillary forces causing a packing together of hemi-hydrate particles.

Work I have carried out on several commercial plasters has shown that crystalline dihydrate appears in measurable quantity before the initial set. It seems likely that the initial thickening of a plaster slurry is due to the formation of this small amount of dihydrate crystals, and therefore the complete setting process, both initial and final, is one of crystallization.

Samples of gauged plaster were removed from the mixing bowl at various times before the initial set, which was determined as described in the Standards Association of Australia Interim Specification No. $317^{3}$. These were washed with ethyl alcohol and 